\title{
ASTRONOMISCHE NACHRICHTEN
}

Band I7r.

Nr. 4086.

\section{Bemerkungen zu den Schlüssen, welche Herr Ch. Lane Poor aus den neueren Messungen des Sonnendurchmessers gezogen hat. \\ Von L. Ambronn.}

In Nr. 2 und Nr. 5 des XXII. Bandes des Astrophysical Journals hat Herr Charles Lane Poor Untersuchungen ther die Gestalt der Sonne veröffentlicht, welche ihn zu der Annabme zu berechtigen schienen, daß der Unterschied zwischen polarem und äquatorealem Durchmesser gewissen, periodischen Schwankungen unterworfen sei. Nachdem Herrn Poor die Ausmessung und Diskussion einer Anzahl älterer Sonnenphotographien ein Resultat ergeben hatte, welches solche Schwankungen zu bestätigen schien, hat er auch die in den Jahren $1872-1884$ mit den kleinen Fraunhoferschen Heliometern gewonnenen Resultate und in Nr. 5 l. c. auch die von Professor Schur und mir ausgeführten Heliometermessungen uber die Größe und Gestalt der Sonne ${ }^{1}$ ) in den Bereich seiner Untersuchungen gezogen. Namentlich der letztere Umstand ist es, welcher mich veranlaßt, auf die in den genannten Schriften mitgeteilten Daten etwas näher einzugehen, zumal Herr Poor einige die Diskussion einigermaßen beeinflussende Fehler in meiner Abhandlung gefunden zu haben glaubt. Bevor ich auf die Resultate weiter eingehe, möchte ich erst diese $\gg$ Fehlerangelegenheit « richtig stellen. Der Mittelwert von $(p-a)$ für 1891, wie ihn die von mir allein zur Diskussion benutzte Tabelle auf Seite 44 meiner Abhandlung enthält, ist tatsächlich richtig, von Prof. Schur abgeleitet worden, nur ist leider für die Beobachtung am 10. und II. September in der Gesamtzusammenstellung für die Differenz $(p-a)-0.04$ statt -0.64 gedruckt worden. Wenn sich bei einer Nachrechnung fremder Werte solche Unterschiede ergeben, so sollte doch immer, bevor ohne Rate gezogen werden, was für Herrn Poor sehr einfach gewesen wäre, denn alle nötigen Daten sind auf Seite 74 abgedruckt. Dort steht: Polar-Durchm. 47.6964, ÄquatorealDurchm. 47.7 28 , Differenz also -164 Einheiten, was mit dem Skalenwert von nahe $40^{\prime \prime}-0.64$ ergibt. Da Prof. Schur bis zu seinem Tode seine Sonnenmessungen, soweit die Reduktionen möglich waren, mit der denkbar größten Sorgfalt auf dem Laufenden erhalten und schon in Tabellen eingereiht hatte, habe ich natürlich nicht jede einzelne Zahl einer Neuberechnung für bedürftig gehalten. Die von mir zur Diskussion weiteres auf Rechenfehler. geschlossen wird, das Original zu

benutzte $\mathrm{Zahl}$ ist also richtig. Der zweite angeführte $\curvearrowright$ Fehler « ist, wie auf den ersten Blick zu sehen, ein einfacher, leider übersehener Druckfehler auf Seite I IO; in der Tabelle auf Seite 44 steht die richtige Zahl, -der $\gg$ Fehler hat also ebenfalls auf die Diskussion keinen Einfluß. Mit dem Fehler für das Mittel vom Jahre 1898 steht die Sache leider anders, dort muß tatsächlich +O". I I statt - O". I I stehen. Eine Vergleichung der Originale zeigt, daß Prof. Schur wohl auch - o." I I geschrieben hatte und diese Zahl von mir mit übernommen worden ist (die $\mathrm{Zahl}$ ist im Original über eine Bleifederzahl mit Tinte darüber geschrieben), obgleich ich eigentlich den Fehler hätte sehen müssen. Dieser Umstand ist sehr bedauerlich und das um so mehr, da alle vier angeführten »Fehler» sich auf die Rechnung Schurs beziehen. Der vierte "Fehler» ist aber wiederum nur dadurch entstanden, daß ich bei der Schlußzusammenstellung die einzelne Beobachtung von 1901, wie es wohl auch richtig sein dürfte, bei der Mittelbildung für r 900 mit berücksichtigt habe, Herr Poor aber nicht. Dies mag zur Klarstellung der gefundenen * Fehler * dienen. Eine Neureduktion der von mir mitgeteilten Mittelzahlen könnte sich also nur auf den Wert von $(p-a)$ für $\mathrm{I} 898$ beziehen.

Die von Herrn Poor nachgewiesene Veränderlichkeit der Sonnenfigur auf Grund der alten Photographien mag hier außer Betracht bleiben, da er wohl selbst kaum annehmen kann, daß der Genauigkeitsgrad, mit welchen die Messungen der Natur der Sache nach haben ausgeführt werden können, Größen erkennen läßt, deren vielleicht vorhandene Realität nicht ohne weiteres bestritten werden kann, die aber ihren numerischen Werten nach gewiß noch kaum den achten oder zehnten Teil von der Unsicherheit erreichen werden, welche den Resultaten aus den genannten Photographien innewohnt. Zwei Zahlen aber bin ich in der Lage näher zu betrachten, nämlich die aus dem Jahre I 894 stammenden ${ }^{2}$ ). Herr Poor gibt auf Seite I 12 für dieses Jahr und die Tage Juli ro und Juli 17 Resultate, welche auf Sonnenphotographien aus jener Zeit beruhen.

Man erhält die folgenden Zahlen (Pol.-D. - Äqu.-D.):

$$
\begin{aligned}
& \begin{array}{l|l|l|c}
\text { Ch. L. Poor } & \text { Schur } & \text { Ambronn } & \begin{array}{c}
\text { Mittel } \\
\text { (Schur u. Ambronn) }
\end{array}
\end{array} \\
& \begin{array}{ll|ll|lll|l}
\text { Juli } 10 & -0.72 \pm 0.24 & \text { Juli } 5 / 6 & +0.15 & \text { Juli } 6 / 7 & -0.25 & -0.05
\end{array} \\
& \begin{array}{ll|ll|lll|} 
& 17+0.36 \pm 0.23 & \# 23 / 24 & -0.25 & * 24 / 25 & -0.04 & -0.14
\end{array}
\end{aligned}
$$

1) Astronomische Mitteilungen der Kgl. Sternwarte zu Göttingen, Teil 7, Ig05.

2) Leider sind aus dem Jahre 1893 keine Messungen mit dem Göttinger Heliometer vorhanden, welche sich eng genug an die von Herrn Poor aus diesem Jahre beigebrachten Daten anschließen. 
Schur hat am 5./6. und am 23./24. Juli gemessen und die in zweiter Linie gegebenen Werte gefunden. Ich habe am 6./7. und am 24./25. Juli gemessen und die in dritter Reihe gegebenen Werte gefunden. Diese kleine Vergleichung zeigt, daß bei Berlicksichtigung der Unsicherheit, welche selbst einer Heliometermessung der Differenz von $(p-a)$ innewohnt (nämlich etwa rund \pm 0.28 ), von einer Schwankung des Wertes $(p-a)$ während dieser Zeit in dem aus den Photographien gefundenen Betrage gar nicht im entferntesten die Rede sein kann. Herr Poor mißt aber in seinen Kurven auf Seite II 3 gerade dieser Epoche, wegen ihrer schönen Parallelität zu der Sonnenfleckenkurve, besonderes Gewicht bei. Auf die ebenfalls in dem ersten Teil der Schrift des Herrn Poor erfolgte Neubearbeitung der Sonnenmessungen, die bei Gelegenheit der Venusvorubergänge erhalten wurden, möchte ich nicht des weiteren eingehen, da vielleicht von kompetenterer Seite geantwortet wird und ich außerdem absolut sicher bin, daß Herr Auwers gerade diesen Untersuchungen die größte Aufmerksamkeit gewidmet hat. Aber darauf möchte ich allerdings doch aufmerksam machen, daß bei einer genauen Betrachtung der Werte der Tabellen auf den Seiten I Io und I I I des XXII. Bandes des Astrophysical Journals von einer Periodizität, wie sie Herr Poor für seine Annahme daraus abzuleiten sich für berechtigt hält, doch wohl kaum die Rede sein kann. Man braucht nur aus der Tabelle auf Seite 1 1 0 die Daten für einzelne Beobachter ohne vorgefaßte Meinung herauszuschreiben, um zu sehen, daß der Verlauf der Werte von $(p-a)$ für die verschiedenen Beobachter durchaus nicht übereinstimmt. Die nachfolgende Tabelle läßt das erkennen.

\begin{tabular}{|c|c|c|c|c|c|c|c|c|}
\hline \multicolumn{3}{|c|}{ Datum } & Schur & Franz & Kobold & Peter & Hartwig & Küstner \\
\hline \multirow[t]{2}{*}{ I $88 \mathrm{I}$} & Sept. & 30 & - & - -. .5 I & - & - & - & - \\
\hline & Nov. & 10 & +0.22 & - & - & - & - & - \\
\hline \multirow[t]{12}{*}{ I 882} & März & I 4 & - & - & +0.28 & -- & - & - \\
\hline & & 25 & - & - & - & +0.06 & - & - \\
\hline & April & 4 & - & - & -0.05 & - & - & - \\
\hline & $\gg$ & 15 & - & - & - & - & - & 0."०0 \\
\hline & Mai & r 5 & - & - & - & - & +0.09 & - \\
\hline & $\gg$ & I 5 & +0.13 & - & - & - & - & - \\
\hline & & 22 & - & +0.06 & - & - & - & - \\
\hline & Nov. & 20 & - & -0.06 & - & - & 一 & - \\
\hline & 》 & 25 & - & - & - & - & - & +0.13 \\
\hline & $\gg$ & 25 & - & - & $-0.0 \mathrm{I}$ & - & - & - \\
\hline & Dez. & 4 & - & - & - & +0.15 & - & - \\
\hline & $"$ & 5 & - & - & - & - & +0.30 & - \\
\hline 1883 & Juni & 4 & - & - & - & - & -0.02 & - \\
\hline
\end{tabular}

Wenn dabei berücksichtigt wird, daß der mittl. Fehler einer jeden solchen Zahl, selbst wenn sie mit hohem Gewicht behaftet sein mag, auf etwa \pm 0.08 angesetzt werden kann, so wird es jedenfalls schwer sein, eine regelmäßjige $A b$ - oder Zunahme der Beträge für $(p-a)$ ausfindig zu machen.

Wenn ich nunmehr auf des Herrn Poor Neureduktion meiner Bearbeitung der Göttinger Beobachtungen zurïckkomme, so bleibt also mit Ausnahme des Mittelwertes für das Jahr 1898 die Tabelle auf Seite 44 meiner Abhandlung bestehen, sie wird daher jetzt lauten:

\begin{tabular}{lccc} 
Jahr & $\begin{array}{c}\text { Schur } \\
\text { 1890 }\end{array}$ & $\begin{array}{c}\text { Ambronn } \\
+0.113\end{array}$ & $\begin{array}{c}\text { Mittel } \\
+0.112\end{array}$ \\
+0.12 \\
1891 & +0.02 & +0.14 & +0.08 \\
\hline I892 & -0.06 & +0.07 & 0.00 \\
1893 & -0.09 & -0.01 & -0.06 \\
1894 & +0.10 & -0.07 & +0.02 \\
1895 & +0.04 & +0.03 & +0.04 \\
1896 & -0.05 & -0.01 & -0.03 \\
1897 & +0.02 & -0.04 & -0.01 \\
1898 & +0.11 & +0.07 & +0.09 \\
1899 & +0.05 & -0.03 & +0.01 \\
I900 & +0.04 & +0.01 & +0.02 \\
1901 & - & +0.06 & - \\
1902 & - & -0.06 & -
\end{tabular}

Die hier allein zur Diskussion heranzuziehenden Mittelwerte der Jahre von 1892 an (denn gerade für die vorliegende Untersuchung müssen die früheren sicher ausgeschlossen bleiben) sind dann

$$
\begin{array}{ccc}
\text { Schur } & \text { Ambronn } & \text { Mittel } \\
\text { ○.०18 } & 0.002 & 0.010 .
\end{array}
$$

Von einer besseren Übereinstimmung dieser Werte als der von mir im Original gegebenen kann doch wohl nicht die Rede sein, denn hier ist die Differenz zwischen Schur und Ambronn 0."or6 und dort 0."009. Herr Poor kann doch wohl nicht bei seiner Vergleichung die Mittelwerte aus r 889-r 900 und die aus 189 r-1900 gemeint haben, welche tatsächlich liefern:

$\begin{array}{cccc} & \text { Schur } & \text { Ambronn } & \text { Mittel } \\ 1889-1902 & +0.028 & +0.022 & +0.025 \\ 1891-1902 & +0.018 & +0.002 & +0.010\end{array}$

denn hier kann man doch die erste Zeile nicht mit der $z$ weiten in unserem Sinne vergleichen, weil beide ja aus denselben Zahlen entstanden, nur sind in der ersten Zeile zwei Reihen enthalten, welche für unsere Zwecke nicht in Vergleich gesetzt werden können. Die bessere Übereinstimmung der beiden Werte für Schur ist aber selbstverständlich, da eben aus dem - O"II ein +o"II geworden 
ist. Ich möchte übrigens, um zu zeigen, wie wenig grade auf die hier benutzten Mittelzahlen Wert zu legen ist, noch die chronologische Zusammenstellung der Werte fur $(p-a)$ fur beide Beobachter für das Jahr 1898 geben; dieselbe wird leicht erkennen lassen, daß der verhältnismäßig große Mittelwert von +o". I I wohl irgend einem rein zufälligen persönlichen Faktor zugeschrieben werden muß, wie das z. B. auch bei der großen Jahresabweichung für 1895 bei meinen Werten für den Sonnendurchmesser selbst der Fall ist (vgl. dazu Seite 42 ff. meiner Abhandlung).

Chronologische Zusammenstellung der Einzelwerte von $(p-a)$ und der Relativzahlen für jeden Tag.

\begin{tabular}{|c|c|c|c|c|}
\hline Beob. & $189^{8}$ & & $(p-a)$ & $\begin{array}{l}\text { Relativ- } \\
\text { zahlen }^{1} \text { ) }\end{array}$ \\
\hline A & Jan. & 8 & -0.120 & I 3 \\
\hline A & 》 & I 4 & +0.30 & 23 \\
\hline A & $»$ & 19 & +0.08 & 65 \\
\hline A & Febr. & 25 & +0.25 & $x 6$ \\
\hline $\mathbf{S}$ & $\triangleright$ & 26 & -0.13 & 24 \\
\hline $\mathbf{S}$ & März & 12 & +0.35 & 82 \\
\hline S & 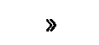 & 2 I & +0.25 & ( 12 2) \\
\hline A & » & 29 & +0.40 & 7 \\
\hline$S$ & April & 6 & -0.20 & 26 \\
\hline A & $\gg$ & 9 & +0.11 & 27 \\
\hline $\mathbf{S}$ & Mai & 2 & +0.05 & 29 \\
\hline A & $\gg$ & 5 & -0.28 & 9 \\
\hline $\mathbf{S}$ & 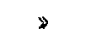 & 14 & +0.30 & $3^{2}$ \\
\hline S & $\triangleright$ & 23 & +0.62 & I 7 \\
\hline A & Juni & 4 & -0.14 & 23 \\
\hline S & 》 & 8 & +0.24 & 2 I \\
\hline A & 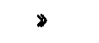 & IO & -0.22 & (4) \\
\hline$S$ & $>$ & I I & +0.29 & 8 \\
\hline $\mathbf{S}$ & $\gg$ & 15 & +0.04 & (18) \\
\hline A & » & 18 & 0.00 & 34 \\
\hline A & $»$ & 24 & -0.06 & I 5 \\
\hline $\mathbf{S}$ & 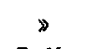 & 27 & -0.23 & 31 \\
\hline A & Juli & 15 & +0.19 & 8 \\
\hline $\mathbf{S}$ & $»$ & 16 & +0.16 & I I \\
\hline A & $»$ & 26 & 0.00 & I 7 \\
\hline $\mathbf{S}$ & 》 & 27 & +0.26 & 7 \\
\hline $\mathbf{A}$ & Aug. & 2 & -0.04 & 65 \\
\hline $\mathbf{S}$ & , & 3 & +0.25 & $(62)$ \\
\hline $\mathbf{S}$ & $》$ & 13 & -0.27 & 49 \\
\hline $\mathbf{S}$ & » & $x_{5}$ & +0.18 & 40 \\
\hline A & $\gg$ & 16 & -0.18 & 29 \\
\hline A & 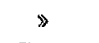 & 22 & +0.29 & I 7 \\
\hline A & Sept. & 7 & -0.04 & 60 \\
\hline A & ; & 14 & +0.38 & 54 \\
\hline $\mathbf{S}$ & Nov. & 2 & -0.05 & 61 \\
\hline A & $\gg$ & 8 & +0.27 & (50) \\
\hline$S$ & 》 & 9 & -0.04 & 50 \\
\hline A & $\gg$ & 18 & +0.26 & (1 2) \\
\hline $\mathbf{S}$ & $»$ & 19 & -0.25 & (1 2) \\
\hline $\mathbf{S}$ & $\gg$ & 23 & -0.18 & (1 3) \\
\hline $\mathbf{S}$ & Dez. & 7 & +0.71 & (25) \\
\hline A & $\gg$ & 22 & +0.06 & 7 \\
\hline
\end{tabular}

Aus dieser Zusammenstellung geht meiner Ansicht nach mehrererlei hervor, und zwar: I) Die Zahl +o". I I für Schur hängt im wesentlichen von einer kurzen Periode im Mai und Juni und von dem Wert dieser Differenz am 7 . Dezember ab. Da Schur häufiger ohne Rücksicht auf die Zwischenzeiten mehrere Beobachtungen dicht zusammenlegte (vergl. die Bemerkung auf Seite 5 I meiner Abhandlung), so können auch leichter zufällige Einflüsse eine Gruppe solcher Werte betreffen. 2) Der Wert von $p-a=+0.62$ am 23. Mai ist durch diejenigen von Mai $5, \mathrm{I}_{4}$ und Juni 4,8 und Io durchaus nicht bestätigt. 3) Der Wert vom 7. Dezember, der, wie Herr Poor selbst gesehen haben wird, ganz aus der Reihe herausfällt, gehört tatsächlich einer Messung an, die bei ganz schlechtem Wetter angestellt worden ist, sie trägt die Bewertung 4, 4, 4 für Ruhe «, \$Schärfe und \Bläue k, d. h. milchiger Hintergrund mit schlecht begrenztem Bilde, und sie wäre vielleicht überhaupt besser weggeblieben. Da ich aber gerade bei nicht eigenen Beobachtungen sehr vorsichtig mit dem Ausschließen sein wollte, blieb sie erhalten. 4) Die beiden Zahlen +o". I und +0. ". 7 , welche sich als die arithmetischen Mittel für 1898 für die resp. Beobachter in der Tabelle auf Seite 44 finden, sagen über den wahren Verlauf der Differenzen von $(p-a)$ in diesem Jahre garnichts aus. Die Zeichenfolge in den Werten von $(p-a)$ ist eine so wechselnde, die absoluten Werte von $(p-a)$ sind so schwankend, daß ein periodischer Verlauf derselben während dieser Zeit ganz ausgeschlossen sein dürfte. Wollte man etwa die wirklich an den einzelnen Tagen gezählten Sonnenflecken mit dem Verlauf in Beziehung setzen, so kommt man zu denselben Resultaten, wie sie die von mir für das merkwürdige Jahr 1895 gegebene Tabelle und Kurve aufweist, die auch nicht den geringsten Zusammenhang zwischen den Abweichungen der Schurschen Durchmesserwerte und meinen Zahlen erkennen lassen mit Bezug auf die aus den Relativzahlen zu folgernde Sonnentätigkeit.

Die Zahlen, welche Herr Poor als mittlere Fehler für die in seiner Tabelle auf Seite 309 gegebenen Daten anführt, sind aber gar nicht die diesen Zahlen zukommenden mittleren Fehler, sondern die mittleren Fehler der Schlußwerte für die Sonnendurchmesser selbst, sie müßten erst wieder mit $\sqrt{2}$ multipliziert werden und dann wird daraus

$$
\pm 0.02 \text { I statt } \pm 0.015 \text { und } \pm 0.013 \text { statt } \pm 0.009
$$

also Werte, die die der wirklich vergleichbaren Mittel noch übersteigen. Das Vertrauen, welches den zur Mittelbildung von $(p-a)$ benutzten Jahresmitteln auf Seite 3 Io innewohnt, wird charakterisiert werden, wenn man den mittleren Fehler eines einzelnen Wertes von $(p-a)$ - für Schur \pm 0.28 , für Ambronn 土0".24 - mit der Quadratwurzel aus der mittleren Anzahl der Jahresbeobachtungen, das sind etwa 16 , also mit 4 dividiert. Das liefert für Schur als mittl. Fehler eines Jahresmittels von $(p-a) \pm 0 . " \circ 7$ und für Ambronn \pm 0.06 . Vergleicht man damit die Zahlen der Tabelle IV, so wird man, ganz abgesehen von den oben geltend gemachten Umständen, den Jahresmitteln ihre wahre Bedeutung leichter beimessen können und einsehen, daß Spekulationen,

1) Diese Rubrik enthält die Relativzahlen für die Sonnentätigkeit nach Professor Wolfer, die eingeklammerten Zahlen sind so gut als möglich interpoliert. 
die auf Beträge jener Zahlen, die kleiner sind als etwa o." 10 und $\circ . \circ 8$, gegründet sind, jede tatsächliche Berechtigung abgesprochen werden muß. Dasselbe kann man wohl auch von Tabelle VI behaupten. (In allen drei Reihen kommen unter den je 42 Zahlen im Durchschnitt je 18 Zeichenwechsel vor.)

Die Re-Discussion des Herrn Poor hat also in dieser Richtung keine neuen Gesichtspunkte erwiesen, zumal wenn man unter Berücksichtigung des oben Gesagten den geradezu idealen Zeichenwechsel in Tabelle IV durch den einfachen Anblick (besonders in der Reihe für Ambronn und im Mittel) erkennt. Auch die in der Figur I auf Seite 3 I 2 beigebrachten Kurven sagen, soweit es die zweite und dritte betrifft 1), meiner Ansicht nach nichts anderes aus, als daß die beiden Werte von $(p-a)$ für 1890 und $189 \mathrm{I}$, die, wie mehrfach dargetan, aus einer Untersuchung der vorliegenden Art ganz wegbleiben sollten, stark nach der positiven Seite von dem allgemeinen Mittel abweichen; fallen diese beiden ersten Jahre weg, so zeigen die Kurven nur noch Schwankungen, die gar nichts besonderes mehr aufweisen, denn alle $\mathrm{Ab}$ weichungen liegen dann innerhalb der Grenze von \pm 0 ". Io oder gar $\pm 0 . . \circ 8$, also innerhalb der Beträge, welche wie oben gezeigt, den mittleren Fehlern der Jahresmittel von $(p-a)$ etwa entsprechen. Des weiteren dürfte der Verlauf der Vorzeichen in Tafel V (S. 313) doch auch nicht für eine Veränderlichkeit sprechen, da offenbar die Darstellung der Werte durch die Annahme der »variable figure * der Sonne nicht besser geworden ist, wenn das auch durch die Abnahme der Fehlersumme scheinbar der Fall ist; denn diese Abnahme ist nicht durch eine richtigere Darstellung, sondern nur durch eine Rücksichtnahme auf die Werte der Jahre I 890 und I89I erfolgt und hat bewirkt, daß die nunmehr übrig bleibenden Beträge erst recht einen periodischen Verlauf aufweisen, der früher gar nicht vorhanden war. Während der Jahre $189^{\circ}$

Sternwarte Göttingen, r 906 Februar.

bis 1895 liegt diese »variable figure " ganz auf der einen Seite der Sonnenfleckenkurve und während des späteren Verlaufes fast ganz auf der anderen Seite, sie zeigt also offenbar einen anderen Verlauf als jene. Außerdem scheint mir doch auch ganz besonders die gar nicht zu tibersehende Phasenverschiebung zwischen Schurs Werten von $(p-a)$ und den meinigen durchaus nicht derart, daß die Maxima und Minima eine Übereinstimmung erkennen lassen, denn z. B. für die Zeiten zwischen 1892 und 1897 liegen die Schurschen Maxima fast genau auf der Mitte zwischen meinen größten und kleinsten Werten von $(p-a)$, so daß es also dem jeweiligen Belieben überlassen bleibt, eine Koinzidenz oder gerade das Gegenteil anzunehmen.

Schließlich möchte ich nur noch hinzufügèn, daß alle diese Fragen über etwaige Periodizität in den Werten des Sonnendurchmessers selbst und derjenigen von $(p-a)$ und Koinzidenz derselben mit anderen ähnlichen Erscheinungen von mir, ich kann wohl sagen jahrelang erwogen worden sind und durch sehr zahlreiche Zusammenstellungen gepruft wurden. Aber auf Grund aller dieser Betrachtungen bin ich zu dem Entschluß gekommen, auf keinen Fall in die Resultate der Göttinger Beobachtungen etwas hineinzutragen, was meiner Überzeugung nach durch dieselben nicht erwiesen werden kann, denn solche billigen Hypothesen bestehen heute, und morgen sind sie durch eine bessere Reihe über den Haufen geworfen. Gerade solche weiteren Reihen arzuregen oder, wenn sie schon vorhanden sind, ihre Publikation zu beschleunigen, halte ich für das richtigere, bevor man aus Werten, die doch immerhin nur mit ein und demselben Instrument gemacht sind und die vielleicht aus ganz anderen, örtlichen oder physiologischen, Gründen fließende Abweichun. gen besitzen können, Schlüsse zieht, deren beweisende Daten innerhalb ihrer eigenen Fehlergrenzen liegen.

\section{Ambronn.}

1) Die Zahlen am Rande der Darstellungen, welche die den Werten von $(p-a)$ entsprechenden Ordinaten abgeben sollen, tragen tibrigens falsches Vorzeichen.

\section{Eclipses totales de Sol.}

Boceto de un aparato para investigar la posición de los elementos productores de las shadow bands (bandas oscilantes) en el espacio.

De Dr. M. Roso de Luna.

Es sabido que momentos antes y después de la totalidad del eclipse de Sol se ven deslizarse por el suelo unas bandas sinuosas, alternativamente claras y obscuras, cuya orientación, dirección de su movimiento, velocidad, etc., se ha tratado de determinar mediante un lienzo blanco tendido horizontalmente en el suelo y orientado de $\mathbf{N}$. á $\mathrm{S}$. Con observaciones asi verificadas, han realizado MM. F. H. Bigelow y W. H. Pickering importantes estudios de síntesis acerca de las causas posibles del fenómeno.

Yo propongo hoy al mundo sabio que se oriente la observación de futuros eclipses en el sentido de buscarse geométricamente la posición en el espacio de los elementos productores de tales bandas, ya sean éstos las diversas corrientes de los vientos, como se cree por muchos, ya se deban á un origen lunisolar, merced á la difracción operada por el borde de la Luna sobre los rayos de la delgada falce solar en las proximidades de la totalidad.

Al efecto de determinar la posición de tales elementos, bastarian tres planos diferentes, $y$ aun dos (vertical y horizontal) con arreglo á las enseñanzas de la Geometría de posición ó descriptiva. Así es como hemos podido nosotros determinar en el eclipse de 30 de Agosto último, en Soria (España), la posición de tales elementos, que resultaron comprendidos en un plano casi vertical (de $88^{\circ}$ á $99^{\circ}$ en 5 segundos), formando con el plano vertical de observación, orientado de $\mathrm{E}$. á W., un diedro menor de $45^{\circ}$. Las bandas se presentan terrososucias muy pobres, con 2 centímetros de anchura y 6 entre banda y banda, ondulantes 6 sinuosas, 